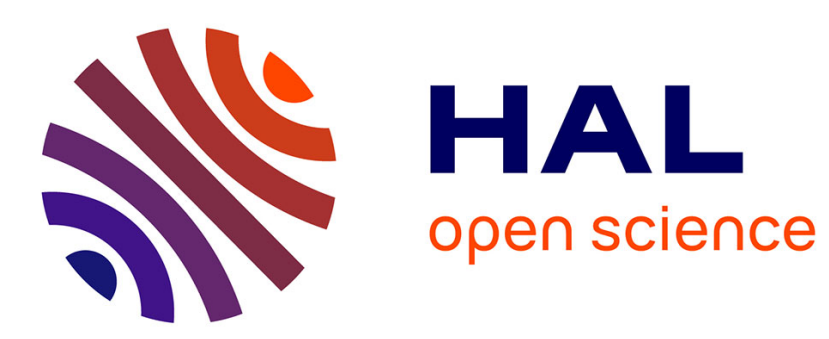

\title{
Navettes domicile-travail : naissance et développement d'un objet statistique structurant
}

\author{
Hadrien Commenges, Julie Fen-Chong
}

\section{To cite this version:}

Hadrien Commenges, Julie Fen-Chong. Navettes domicile-travail : naissance et développement d'un objet statistique structurant. Annales de géographie, 2017, 3 (715), pp.333-355. halshs-01548992

\section{HAL Id: halshs-01548992 \\ https://shs.hal.science/halshs-01548992}

Submitted on 28 Jun 2017

HAL is a multi-disciplinary open access archive for the deposit and dissemination of scientific research documents, whether they are published or not. The documents may come from teaching and research institutions in France or abroad, or from public or private research centers.
L'archive ouverte pluridisciplinaire HAL, est destinée au dépôt et à la diffusion de documents scientifiques de niveau recherche, publiés ou non, émanant des établissements d'enseignement et de recherche français ou étrangers, des laboratoires publics ou privés. 
Commenges H., Fen-Chong J. (2017) "Navettes domicile-travail : naissance et développement d'un objet statistique structurant", Annales de géographie, $n^{\circ} 715$, pp.333-355.

\section{Navettes domicile-travail : naissance et développement d'un objet statistique structurant}

\section{Introduction}

Le nouveau zonage en aires urbaines de l'INSEE, établi à l'aide des navettes domicile-travail, dessine une image de la structuration du territoire français par des pôles urbains, et permet d'apprécier « l'influence des villes au-delà de leur limites physiques définie par la continuité du bâti » (Brutel, 2011). Les navettes « esquissent des espaces de vie et permettent d'observer des relations privilégiées entre des lieux » (Gilli, 2002 : 290). Les navettes permettent d'appréhender « le rôle de la mobilité sur la transformation des espaces du quotidien » (Massot, 1998 : 155) et de dresser la carte des territoires vécus (INSEE et al. 2002). Pour qui ignore ce qu'est une navette, il peut paraître surprenant qu'un même objet statistique permette d'appréhender la structuration des espaces, serve de critère de rattachement à l'espace urbain, soit le marqueur de territoires vécus et le reflet principal des pratiques de mobilité quotidienne.

Ce que l'on désigne sous le nom de «navettes», de « migrations alternantes » ou de « migrations pendulaires » est le lien réalisé entre deux informations renseignées au niveau individuel par le recensement de la population : le lieu de résidence et le lieu de travail. Cette première définition appelle deux précisions importantes. D'une part, les navettes ne concernent qu'une fraction de la population, la population active occupée dont la commune de travail est différente de la commune de résidence, qui représente en 2008 environ le quart de la population française. D'autre part, il faut un certain type de construction empirique et/ou théorique pour produire un discours technique ou scientifique sur la mobilité quotidienne et sur la structuration spatiale à partir des navettes. En effet, en termes de mobilité, l'objet navette ne dit rien sur l'existence, sur la fréquence et sur l'itinéraire du potentiel déplacement entre le domicile et le travail. En termes de structuration spatiale, cet objet ne dit rien sur la morphologie urbaine, sur la structure des réseaux ni sur les pratiques spatiales. Malgré cette apparente limitation, les navettes sont depuis les années 1960 l'objet d'un nombre croissant de travaux, dans les champs de la géographie, de la démographie et de la socioéconomie des transports. 
Nous partons du constat que l'objet navette, depuis sa naissance dans les années 1930, a pris une importance grandissante dans les études de géographie urbaine et de mobilité quotidienne. Alors que cet objet a très peu évolué dans son mode de production, son domaine de validité s'est considérablement étendu. Sur la base de ce constat, bien documenté dans ce travail, nous chercherons d'abord à comprendre les raisons de la montée en importance de l'objet navette, puis nous préciserons les constructions empiriques et théoriques sur lesquelles s'appuie l'extension de son domaine de validité. Nous faisons I'hypothèse que ces constructions ont façonné une certaine vision du territoire et de mobilité que nous qualifierons de «salariale », en écho aux travaux d'Antoine Haumont (2000). La notion de «salariat » est reste assez peu utilisée dans les sciences humaines et sociales, qui mobilisent plutôt la catégorie englobante de « travail » (Vatin et Bernard, 2007). Pourtant le développement du salariat est étroitement lié à la structuration des espaces urbains par des flux massifs et hiérarchisés reliant des lieux de résidence et des lieux d'emploi. Qui plus est, la naissance de l'objet statistique navette est issue de l'émergence d'une société majoritairement salariale. Notre article prétend réhabiliter cette notion de salariat, que seul Antoine Haumont a réellement utilisé pour appréhender les mobilités quotidiennes, en tous cas dans le cas français.

Cette analyse concerne la littérature scientifique et technique française, cependant un certain nombre d'assertions seraient aisément transposables à d'autres pays, en particulier du fait de la circulation des savoirs techniques après la Seconde Guerre Mondiale (Gardon et al., 2009). La navette est devenue au cours du XXe siècle l'objet qui traduit la structuration de l'espace, qui participe à la définition de son urbanité, qui résume les pratiques de mobilité quotidienne et dessine les espaces de vie. A sa création, elle n'était pourtant destinée à aucune de ces tâches. Comment l'objet navette, dont le pouvoir descriptif et explicatif de la structuration de l'espace et de la mobilité quotidienne est apparemment limité, en est arrivé à occuper une situation de quasi-monopole en cette matière ?

\section{L'effet lampadaire}

Le processus de construction de l'information quantitative fait l'objet de travaux dans des domaines variés : les catégories socio-professionnelles (Desrosières et Thévenot, 1979), les catégories ethniques (Schor, 2001) ou encore la statistique agricole (Didier, 2009). Tous ces travaux ont en commun de considérer que les dispositifs statistiques ne sont pas neutres ni transparents, qu'ils ne sont pas de simples leviers au service de l'action publique, mais qu'ils contribuent à structurer le contenu de cette action et en façonner les contours.

Dans ce positionnement commun face aux dispositifs statistiques, deux idées méritent une attention particulière : l'idée de chaîne de production des données d'une part, et le processus de co-construction des phénomènes et de leur mesure d'autre part. L'idée d'une chaîne de production des données est présente dans les premiers travaux de socio-histoire de la statistique (Affichard, 1977a ; 1977b), mais c'est sans doute dans l'ouvrage d'Emmanuel Didier (2009) qu'apparaît le plus 
clairement sa matérialité. Dans cet ouvrage il s'attache à suivre pas à pas, de bout en bout, la chaîne de production des statistiques agricoles américaines et montre comment le produit final est façonné par chacune des étapes de la chaîne. Quant au processus de co-construction, il consiste à considérer qu'il n'y a pas de dispositif statistique exogène intervenant a posteriori pour saisir un phénomène préconceptualisé, mais un processus de co-construction du phénomène et du dispositif statistique qui le mesure (Desrosières et Thévenot, 1979 ; 1988). Ces deux idées permettent de mieux comprendre la nature et le fonctionnement des dispositifs statistiques, mais elles n'expliquent pas comment ces dispositifs se maintiennent au cours du temps malgré l'évolution des techniques de production et d'analyse des données et malgré l'évolution des questions susceptibles d'être posées. La notion de chaîne de production des données doit ainsi être enrichie d'un mécanisme expliquant sa stabilité au cours du temps.

Immanuel Wallerstein (2009 : 38) formule de façon imagée I'injonction suivante : il ne faut pas, «comme l'ivrogne qui cherche sa clé perdue sous le lampadaire parce que c'est là qu'il y a de la lumière », choisir le problème en fonction des données disponibles, mais au contraire chercher ou produire les données en fonction du problème. Cette injonction, qui a toute l'apparence du bon sens, véhicule en fait l'image d'un processus de production des données qui partirait perpétuellement d'un état initial vierge de données, vierge de questions et vierge de contraintes et de pratiques organisationnelles : un reset à chaque nouvelle édition. Pourtant, à chaque instant, les personnes et les organisations cherchant à produire une connaissance se placent dans un périmètre éclairé par les dispositifs statistiques existants, par les pratiques et les résultats antérieurs. En filant la métaphore précédente, il faut insister non sur l'apparente absurdité du comportement de l'ivrogne, mais sur l'interaction entre ce comportement et le contexte qui le produit. II en est de même pour les dispositifs statistiques et les recherches qu'ils nourrissent, ces deux éléments interagissent : les dispositifs statistiques contraignent et façonnent certaines conceptions et certains questionnements, ces conceptions et ces questionnements nourrissent et justifient en retour l'existence du dispositif. Ainsi, la chaîne de production et d'utilisation des données se maintient et se consolide par un mécanisme d'auto-renforcement. Cette idée est une application de la notion de processus dépendant au chemin (path dependent) mobilisée en économie (Arrow, 2000) et en sciences politiques (Pierson, 2000). Cette dépendance n'est pas déterministe mais elle canalise et restreint l'éventail de choix et lie chaque étape du processus aux étapes antérieures.

Trois types de mécanismes se dégagent qui marquent le chemin et consolide la dépendance. D'abord, l'éventail de choix est restreint par les données disponibles. Ensuite, la chaîne de production et d'utilisation des données est canalisée par la volonté de capitaliser l'acquis des travaux antérieurs. Dans le cas d'études quantifiées, cet acquis repose sur des dispositifs statistiques dont la pérennité garantit une comparabilité dans le temps. Enfin, l'éventail de choix est canalisé par l'effet lampadaire du dispositif statistique. Celui-ci, par son ancrage dans le paysage technique et scientifique canalise les questions d'intérêt.

Le premier mécanisme - la disponibilité des données - est fréquemment invoqué dans le cas des navettes domicile-travail. Par exemple, P. Bertrand et J. Hallaire 
(1962 : 186) indiquent que « les migrations alternantes ne se limitent pas aux mouvements pendulaires de la population active [...] néanmoins on se limite généralement, surtout pour des raisons de facilité d'observation statistique, aux migrations alternantes des actifs ». Pierre Merlin (1967 : 247) consacre un chapitre de sa thèse d'État aux déplacements autres que les migrations alternantes et commence par constater que «malheureusement les sources sont médiocres ». Ce mécanisme de maintien n'est pas spécifique aux navettes domicile-travail et ne fera donc pas l'objet ici d'une étude approfondie.

Le deuxième mécanisme - la nécessité de comparabilité dans le temps - est très souvent explicité lorsque le dispositif statistique concerné fait l'objet d'une critique de fond qui menace son existence. Dans le cas des navettes, l'existence du dispositif n'a jamais été menacée et ce potentiel mécanisme de maintien n'a donc jamais été mis en avant.

Le troisième mécanisme - l'effet lampadaire - est au cœur de notre travail. La thèse de cet article est que la montée en force de l'objet navette vient du renforcement mutuel des objets d'intérêt : les navettes et l'espace urbain. D'abord, les déplacements domicile-travail ont occupé une place grandissante dans les études urbaines parce qu'ils étaient les plus importants, par leur nombres et surtout par leur rôle structurant. Dès la fin des années 1950, les navettes commencent à être utilisées pour la délimitation des agglomérations urbaines (Brichler, 1958 ; Brichler et Bastié, 1960) et ce type d'utilisation s'est maintenu jusqu'au dernier zonage de I'INSEE (Brutel, 2011). Bref, depuis plus d'un demi-siècle les navettes contribuent à définir l'urbain et à donner une image de la mobilité quotidienne dans les espaces urbains. En parallèle, l'espace urbain a occupé une place grandissante dans les études de mobilité et de transport parce que c'est dans cet espace que se sont cristallisés les problèmes liés à la circulation (Dupuy, 1975 ; Gardon, 2009). Depuis les années 1960, la grande majorité des études de mobilité quotidienne s'intéresse aux milieux urbain et périurbain. On assiste ainsi à un renforcement mutuel des objets d'intérêt : les navettes occupent une place grandissante dans les études urbaines, l'espace urbain occupe une place grandissante dans les études de mobilité, qui s'appuient souvent sur les navettes.

\section{Corpus d'archives et d'entretiens}

On retrouve dans les travaux sur les navettes deux postulats qui méritent d'être discutés : le premier est que les navettes permettent de saisir les comportements de mobilité quotidienne et les espaces de vie (Gilli, 2002 ; Hilal et Sencébé, 2003 ; Berger, 2004), le second est que les navettes structurent l'espace et en particulier l'espace urbain (Berroir, 1998 ; Berroir et al., 2004 ; 2007). Nous avons construit un corpus d'archives et d'entretiens permettant une analyse historique de ces deux postulats, en réunissant deux ensembles de matériaux produits dans des cadres différents. Le corpus résultant est certes composite mais riche de complémentarités (cf. Figure 1). D'abord, l'objectif originel de production de chacun des deux souscorpus recoupe les deux postulats précités : le premier sous-corpus d'archives et d'entretiens a été produit dans le cadre d'un travail sur l'histoire de la socio- 
économie des transports, et plus généralement sur les dispositifs de mesure de la mobilité quotidienne ; le second a été produit dans le cadre d'un travail sur l'histoire des zonages de I'INSEE. Ensuite, ces deux sous-corpus appréhendent l'objet navette à travers deux prismes temporels complémentaires : le premier amène une vision sur le temps long, qui remonte à la fin du XIXe siècle, alors que le second fait un zoom sur le travail de zonage de I'INSEE durant les années 1990. La fusion des deux permet ainsi une vision d'ensemble de l'utilisation de l'objet navette de ses origines à nos jours, couplée à une étude de détail du travail de zonage de l'INSEE sur la base des navettes.

\section{Figure 1. Corpus d'archives et d'entretiens}

Pratiques de mobilité quotidienne
Période couverte : 1960 - 1990
CORPUS D'ARCHIVES
Institut National de la Statistique et des Études Économiques (Insee)
Institut National des Études Démographiques (INED)
Service des Études Techniques des Routes et des Autoroutes (SETRA)
Institut d'Aménagement et d'Urbanisme d'île-de-France (IAU-IdF)
Centre de documentation du ministère du Développement Durable (CRDD)
CORPUS D'ENTRETIENS
Daniel Courgeau
Serge Goldberg
Jacques Lesourne
Pierre Merlin
Christophe Terrier

Structuration de l'espace urbain
Période couverte : 1993 - 1996
CORPUS D'ARCHIVES
Littérature grise autour de la révision des ZPIU
Comptes-rendus de réunion du groupe de travail
Travaux intermédiaires
Échanges de courrier entre les membres du groupe
CORPUS D'ENTRETIENS
Mohamed Hilal
Philippe Julien
Loeiz Laurent
Thomas le Jeannic
Christophe Terrier
Vincent Vallès

Le premier sous-corpus s'inscrit dans le cadre d'un travail visant à mettre en perspective le contenu des dispositifs actuels de mesure et de modélisation de la mobilité quotidienne, en examinant le contexte de leur création et les conditions de leur maintien malgré l'évolution des techniques et des questions susceptibles d'être posées. En réunissant des matériaux sur les dispositifs de mesure de la mobilité, en particulier sur les dispositifs spécialisés comme les comptages et les enquêtes origine-destination, l'objet navette s'est rapidement imposé comme un objet statistique majeur dans ce domaine. Un ensemble de documents écrits portant une information sur les navettes a été recueilli dans ce cadre, par une recherche systématique dans les archives de I'INSEE, de I'Institut National des Études Démographiques (INED), du Service des Études Techniques des Routes et des Autoroutes (SETRA), de l'Institut d'Aménagement et d'Urbanisme d'île-de-France (IAU-IdF) et du Centre de documentation du ministère du Développement Durable (CRDD). Cet ensemble est hétéroclite par la nature des documents recueillis, allant du bulletin de recensement à l'article de revue scientifique, en passant par le rapport d'étude et la note manuscrite. Le premier sous-corpus d'archives orales est constitué de cinq entretiens avec des personnes ayant joué un rôle clef dans l'étude des navettes, à différentes époques : Jacques Lesourne, Daniel Courgeau, Serge Goldberg, Pierre Merlin, Christophe Terrier. Jacques Lesourne est co-fondateur de la Société d'Économie et de Mathématiques Appliquées (SMA puis SEMA) d'où est 
issue l'une des premières modélisations des déplacements domicile-travail en 1963 (SEMA, 1963). Daniel Courgeau entre à I'INED en 1966, il devient l'une des références majeures dans le domaine de la mesure des mobilités spatiales (Courgeau, 1988). Serge Goldberg est directeur des études générales de I'Institut d'Aménagement et d'Urbanisme de la Région Parisienne (IAURP) de 1960 à 1967. C'est sous son impulsion que l'IAURP développe un savoir-faire sur les transports fondé sur la mesure et la modélisation des déplacements (IAURP, 1966), entre autres des déplacements domicile-travail. Pierre Merlin entre à I'IAURP dans l'équipe de Serge Goldberg en 1964. II construit avec Michel Barbier ce savoir-faire de I'IAURP en matière de mesure et de modélisation des déplacements, en particulier des déplacements domicile-travail (IAURP, 1966 ; Merlin, 1967). Christophe Terrier entre à I'INSEE en 1972 où il réalise le dispositif MIRABELLE permettant d'analyser les navettes et de construire des zonages sur cette base (Terrier, 1980).

La période couverte par ces entretiens va du début des années 1960 au début des années 1980. L'une des questions posée concernait l'origine de l'objet navette : depuis quand existe-t-il une question sur les navettes dans le recensement de la population ? Après retranscription et analyse de ces entretiens, deux constats se sont imposés : le premier est qu'aucune des personnes interrogées n'a pu répondre avec certitude à cette question. Le second constat est qu'elle était mal formulée : il n'y a jamais eu de question sur les navettes dans le recensement, mais plutôt une question sur la localisation du lieu de travail, ce qui est très différent.

Le second sous-corpus s'inscrit dans le cadre d'un travail visant à retracer le processus de création de la carte des territoires vécus réalisée par l'INSEE, l'INRA et la DATAR (2002). L'hypothèse formulée dans ce cadre est que le zonage en aires urbaines n'est pas le résultat d'un simple travail de modélisation mais aussi le fruit de contraintes organisationnelles et administratives. II s'agit en effet d'un processus complexe fait d'interactions entre des individus en poste, des administrations, des dispositifs techniques et des sources d'information. Ce questionnement très précis a rapidement amené à une réflexion plus générale à partir des matériaux recueillis sur les conditions de production des zonages par l'INSEE. II s'agit d'abord d'un ensemble d'entretiens avec des personnes du groupe de travail réuni pour produire la carte des territoires vécus : Christophe Terrier ${ }^{1}$, Vincent Vallès, Loeiz Laurent, Thomas le Jeannic, Mohamed Hilal, Philippe Julien. Tous ont publié des articles, en particulier dans les publications de I'INSEE, pour diffuser les résultats de leur réflexion sur les zonages (INSEE, 1998 ; Vallès et Hugon, 1994 ; Le Jeannic, 1996 ; Hilal et al., 1995 ; Julien 2000).

Ces publications officielles donnent à voir le résultat final mais elles ne permettent donc pas de retracer les discussions et les débats qui ont eu lieu en interne, tout au long de l'élaboration du nouveau zonage. Nous nous intéressons ici au processus de mise en place du zonage et non à son résultat. Pour cela nous nous appuyons sur

$1 \quad$ Christophe Terrier est donc présent dans nos deux sous-corpus d'entretiens : dans le premier, les questions portaient sur l'ensemble de sa carrière à I'INSEE (des années 1970 à nos jours) ; dans le second, les questions se focalisaient sur le travail de zonage de l'INSEE des années 1990. 
un corpus de littérature grise qui a été mise à disposition par Mohamed Hilal. Il avait conservé un vaste ensemble de documents comprenant les comptes-rendus de réunion du groupe de travail, les travaux intermédiaires, et les échanges de courrier entre les membres du groupe. Les auteurs des documents sont souvent les membres du groupe de travail eux-mêmes, mais aussi des membres de l'INSEE affectés dans d'autres services ou dans des directions régionales. Le zonage en aires urbaines et en aires d'emploi de l'espace rural (ZAUER) est étroitement lié au zonage en aires urbaines (ZAU) destiné à remplacer les zones de peuplement industriel et urbain (ZPIU). Les archives conservées par Mohamed Hilal ne se limitent donc pas à la construction du ZAUER, mais englobe plus généralement les discussions autour de la révision des ZPIU.

La période couverte par ce second sous-corpus d'archives et d'entretiens va de 1993 à 1996. Malgré cette courte période de production, les matériaux recueillis condensent plusieurs couches sédimentées de pratiques scientifiques et administratives. En effet, les personnes interrogées, les dispositifs statistiques qu'elles utilisent et les administrations dans lesquelles ces personnes évoluent cumulent ont une histoire dont le poids se fait sentir. C'est précisément cet aspect qui sera mis en avant dans cette approche historique de l'objet navette : comment les personnes, les organisations et les dispositifs techniques s'inscrivent dans un héritage historique qui les contraint ? En quoi les pratiques de zonage et, d'une façon plus générale, l'usage de l'objet navette sont le produit de cet héritage ?

\section{Naissance conjointe des navettes et du salariat}

Considérer que le processus de production et d'utilisation des navettes est dépendant au chemin a deux implications directes : la première est qu'on ne peut comprendre ce qu'est cet objet aujourd'hui sans remonter à ses origines. La deuxième, moins triviale, est que ces origines ont un poids supérieur aux développements les plus récents puisque ce sont elles qui marquent le chemin de dépendance. Lorsque le chemin de dépendance se creuse, les nouveautés ne parviennent à exister qu'en s'y arrimant. Ainsi, au lieu de présenter des alternatives au chemin principal, elles le renforcent. L'histoire qui suit en est une illustration.

Pour retracer la montée en importance de l'objet navette dans les études de géographie, de démographie et de transport, il a donc fallu rechercher son acte de naissance. Les archives de l'INSEE font apparaître une double naissance répondant à deux questions distinctes : depuis quand peut-on travailler sur les navettes et depuis quand travaille-t-on effectivement sur les navettes ? Ces deux questions amènent à s'intéresser à deux époques différentes.

L'objet navette est issu du lien réalisé au niveau individuel entre lieu de résidence et lieu de travail. Le recensement de la population ayant toujours renseigné la commune de résidence des individus, la possibilité d'établir ce lien dépend de l'introduction d'une question portant sur la localisation du lieu de travail. Celle-ci apparaît dans le recensement de 1896, et constitue l'acte de naissance du dispositif de recueil des navettes. Or l'analyse des archives de I'INSEE fait apparaître que le premier document d'étude des navettes date de 1932 (Bunle, 1932). Sachant le 
coût de l'opération de recensement et la parcimonie qui le caractérise, les trente années qui séparent l'introduction de la question sur le lieu de travail et le premier document d'analyse des navettes laissent penser qu'il s'agit d'un objet dérivé d'une question servant un autre but. Pour bien comprendre la nature de la navette, il faut donc revenir à l'origine de la question qui crée cet objet en introduisant une question sur la localisation du lieu de travail. C'est cette question qui marque un lien durable entre statistiques de la population et statistiques du travail.

En France, la pratique de recensement de la population devient systématique avec le recensement de 1801. Le recensement de la population devient alors global dans l'espace, régulier dans le temps et s'appuie sur des méthodes statistiques de plus en plus précises. Dès les premiers recensements réalisés dans la France contemporaine, l'unité spatiale de référence est la commune. Le critère permettant d'assigner une population à une commune a changé à plusieurs reprises jusqu'à aujourd'hui, considérant uniquement la population de droit (population domiciliée dans la commune), uniquement la population de fait (population présente dans la commune le jour du recensement), ou bien une combinaison des deux (Godinot, 2005).

L'introduction d'une question sur la localisation du lieu de travail (cf. Figure 2) demande une explication. Le préambule du recensement de 1896 reste évasif sur ce point : «les renseignements plus détaillés que fournira la nouvelle méthode de recensement des professions serviront de base à l'étude des projets de loi à préparer ultérieurement en vue de satisfaire aux besoins que révéleront les intérêts sociaux et économiques constatés » (SGF, 1899 : 7). Cette phrase doit être comprise dans le cadre de la mise en place d'une administration du travail issue de l'essor du salariat. Ce dispositif administratif est conçu pour «l'observation, la prise en considération du monde du travail, la réglementation, la législation, l'application de celle-ci, son contrôle, la défense, la représentation des intérêts des travailleurs, l'arbitrage et la conciliation » (Moret-Lespinet, 2006 : 19). La mise en place de cette administration se traduit par la création de plusieurs institutions : I'Office du Travail en 1891, le corps de I'Inspection du Travail en 1892 et le ministère éponyme en 1906. Entre 1880 et 1890, tous les pays industrialisés se dotent de bureaux de statistique du travail, préalable nécessaire à la mise en forme d'un État-providence défini par sa capacité à intervenir sur le monde du travail par des lois sur les retraites, sur le chômage, sur la durée de travail, les accidents et les assurances maladies (Desrosières, 2003). 
Figure 2. Bulletin individuel du recensement de la population de 1896

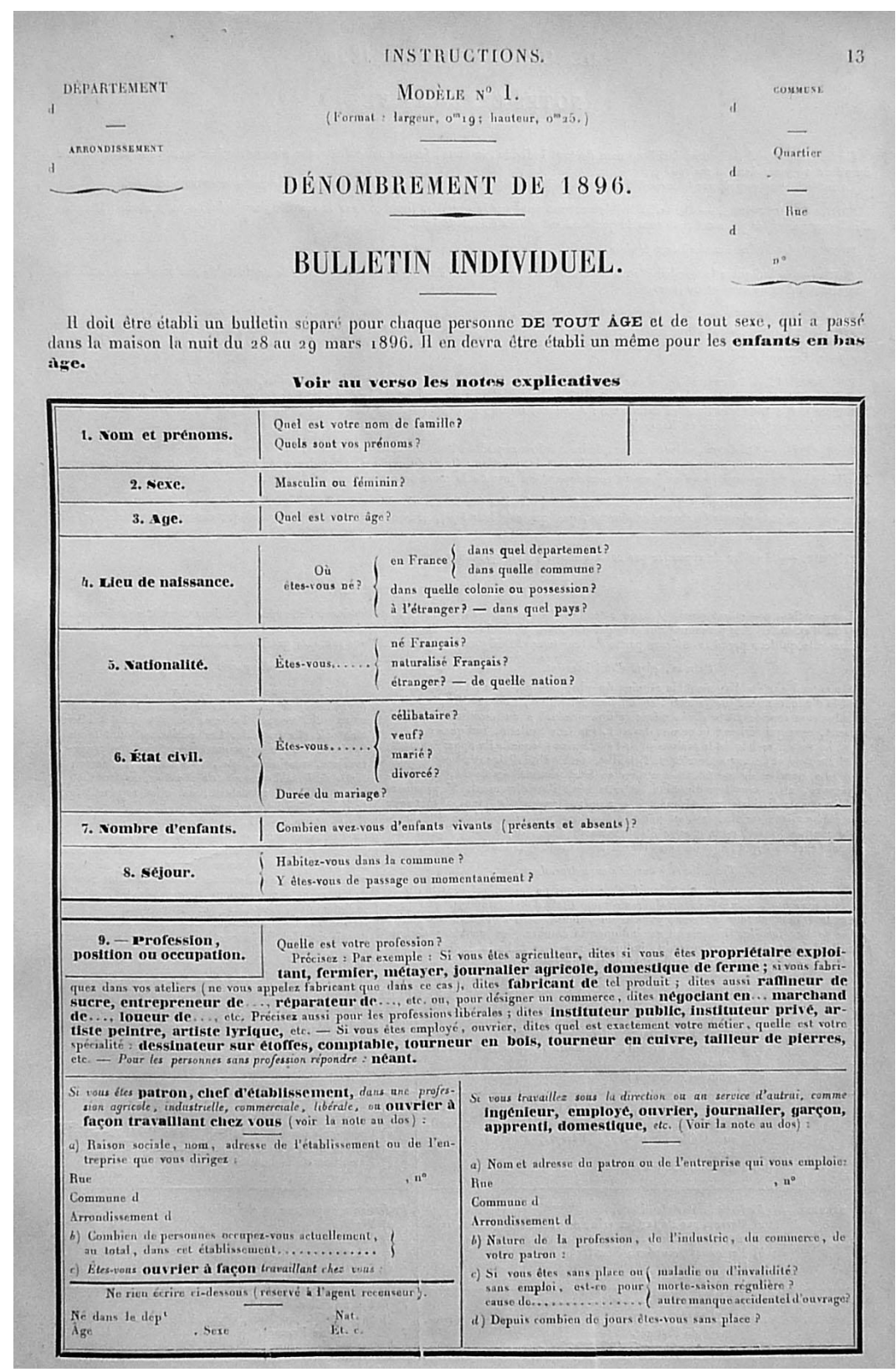

L'objectif de la question sur le lieu de travail introduite au recensement de 1896 est donc de « fournir des indications essentielles sur les intérêts économiques et sociaux du pays et des diverses régions » (SGF, 1899 : 9). Cette statistique du travail naissante s'appuie sur la définition d'une unité de comptage, l'établissement, désignant « tout groupe de personnes travaillant en commun » (Service du Recensement, 1904 : 13). L'établissement est défini par analogie avec le ménage : « le ménage est la communauté d'habitation, l'établissement la communauté de travail »(SGF, 1899 : 9). La question concernant la commune de travail permet de 
réaliser un « classement suivant le domicile de travail » et non suivant le « domicile de résidence ». Ainsi, il est possible de travailler sur les navettes depuis 1896, mais parler d'un objet navette à cette époque est anachronique puisque la question du recensement poursuit des objectifs bien différents. II ne s'agit pas de créer un objet de flux intermunicipal, mais de créer un nouvel objet de stock en assignant la population non plus à la commune de résidence mais à la commune de travail.

Cette dernière remarque revêt une importance capitale pour comprendre l'usage qui sera fait de la question sur le lieu de travail. Les fondements de la statistique du travail s'appuient sur une information renseignée au niveau individuel (raison sociale, adresse et activité de l'établissement): on reconstitue un objet établissement à partir des bulletins individuels (Volle, 1982). II y a eu avant 1896 des essais de recensements industriels spécifiques réalisés non pas au « domicile de résidence » des individus mais au « domicile de travail ». Cette possibilité est écartée car elle ne permet pas de saisir un marché du travail fait à la fois d'établissements industriels mais aussi de petites unités artisanales. Dans un système de production de ce type, les lieux (de domicile et de travail) et les liens (familiaux et professionnels) se confondent, il n'y a pas de distinction nette entre le monde du travail et le monde domestique (Topalov, 1998). La décision de fonder la statistique du travail sur le recensement de la population revêt une grande importance: à partir de ce moment, les statistiques du travail et les statistiques de la population tendent à se confondre.

La question introduite en 1896, qui est la base de la statistique du travail, devient aussi la principale source d'étude de la mobilité à l'échelle des individus et des unités spatiales. Le maillage communal qui s'impose pour alimenter la statistique du travail naissante s'impose comme maillage pour analyser les flux de navetteurs. Pour qu'une certaine mobilité soit appréhendée par ce dispositif statistique il faut que les deux lieux, domicile et travail, soient distincts. En d'autres termes, la mobilité prise en compte est exclusivement intermunicipale. Ce premier point est à la fois la caractéristique principale des migrations alternantes et l'origine de ce terme ${ }^{2}$, traduction littérale de l'allemand Pendelwanderungen (Bunle, 1932). L'usage du terme de migration indique avant tout le passage d'une frontière, un déplacement inter-zones, quelqu'en soit le motif : «les migrations alternantes ne se limitent pas aux mouvements pendulaires de la population active ». C'est le dispositif statistique qui limite le champ des navettes au motif travail « pour des raisons de facilité d'observation statistique » (Bertrand et Hallaire, 1962 : 186). Une migration alternante, ou navette, est donc un déplacement qui implique un franchissement de frontière municipale et un retour dans la même journée.

L'objet navette prend ainsi racine dans le développement de la statistique du travail, corollaire de la consolidation d'une société salariale.

2 Le terme de « navette » est plus récent, c'est Daniel Courgeau qui l'introduit et popularise son usage à la fin des années 1960 (Entretien avec D. Courgeau, Entretien avec Ch. Terrier). 


\section{Le déséquilibre territorial, produit du salariat}

Les premiers travaux sur les navettes datent du début des années 1930, mais c'est à partir des années 1950 que cet objet statistique connaît un développement rapide dans le domaine des études de démographie, d'économie, de géographie et de transport. Cette montée en importance doit être rattachée à l'émergence d'une problématique majeure dans les études territoriales, et plus particulièrement les études urbaines: la problématique du déséquilibre. Dans un contexte de planification rationnelle qui prétend ne rien laisser au hasard (Massé, 1965) on assiste à une co-construction en trois volets : développer un discours sur le déséquilibre, consolider les dispositifs permettant de le mesurer, imaginer des politiques publiques pour lutter contre ses conséquences jugées néfastes.

Le discours sur le déséquilibre s'inscrit dans le cadre d'une réactivation des traditionnelles métaphores organiques et machinistes de la ville (Claval, 1980): « quand une ville est en mauvaise santé, elle connaît la gangrène et la congestion, c'est-à-dire les taudis et les embouteillages » (Pierre Sudreau, cité par Chevalier, 1956 : 633). L'idée de la ville comme organisme malade est couplée avec la rhétorique du déséquilibre, popularisée par Le Corbusier (1971 [1943]) puis par J.-F. Gravier (1947) et largement reprise par les aménageurs de l'après-guerre (Cohen, 2004). La Charte d'Athènes en fait un thème récurrent dans les sections sur le travail, l'habitation et la circulation : « la rupture avec l'ancienne organisation du travail a créé un désordre indicible [...] le grand mal de l'époque actuelle en est issu : le nomadisme des populations ouvrières » (Corbusier, 1971 : 66) ; « les rapports normaux sont rompus entre ces deux fonctions essentielles de la vie: habiter, travailler » (p. 67) ; «le désordre apporté par le machinisme dans un état qui comportait jusque-là une harmonie relative » (p. 96).

Les navettes ne sont pas un objet d'étude en soi, elles sont le symptôme d'autre chose : le déséquilibre dans la localisation des logements et des activités. La plupart des travaux des années 1950 et 1960 traitant des navettes adoptent un angle d'attaque commun, l'étude de la répartition des lieux de résidence et des lieux de travail : « cette étude n'a pas la prétention d'avoir épuisé, sur le plan statistique, tous les aspects de la localisation des lieux de travail et des zones d'habitation dans la région parisienne. En conclusion, nous nous garderons d'exagérer l'importance des déséquilibres géographiques existant entre les uns et les autres. Mais, pour autant que de tels déséquilibres existent effectivement, il importe de les atténuer et surtout d'enrayer leur extension » (Flaus, 1953 : 119). La discussion issue de ce travail sur les navettes, rapportée dans ce numéro du Journal de la Société Statistique de Paris, porte non pas sur les navettes elles-mêmes mais sur le rétablissement de l'équilibre géographique entre les lieux de travail et les zones d'habitation ( $p .120$ ) et sur les expériences de décentralisation industrielle.

Dix ans plus tard, Pierre Merlin entre à I'IAURP et commence sa thèse d'État sur Les transports parisiens (Merlin, 1967). Dans cette thèse, il ouvre la partie sur les migrations alternantes par un chapitre intitulé « Répartition géographique des emplois et des populations, cause des migrations alternantes ». A la même époque, la SEMA construit un modèle alimenté non par les navettes elles-mêmes mais par les localisations des lieux de résidence et des lieux d'emploi (SEMA, 1963). Tous ces 
éléments attestent que l'objet d'étude principal n'est pas la mobilité elle-même, mais le déséquilibre territorial dont les navettes sont le symptôme. Dans la littérature anglo-saxonne, cette problématique du déséquilibre est mise en avant à la même époque par John F. Kain (1968) sous le nom de spatial mismatch hypothesis. D'innombrables travaux de géographie, de sociologie et d'économie s'inscriront dans cette lignée, dont J. F. Kain (2004) a récemment fait la synthèse. Que ce soit dans la littérature francophone ou anglo-saxonne, ce déséquilibre (mismatch) s'applique exclusivement à la répartition des lieux de résidence et des lieux de travail.

Le déséquilibre domicile-travail a pour effet la saturation des réseaux de transport. A la fin des années 1950, le problème des transports prend de l'importance dans le débat public et mène à la création de groupes de travail et de comités spécialisés, comme le Comité National pour l'Aménagement des Horaires de Travail en 1958 (Allain, 2007). Dans les années qui suivent sa création, le CNAT réalise plusieurs études sur les déplacements et les horaires de travail (Maquet, 1963 ; CNAT, 1964). Dix ans plus tard, les événements de mai 1968 véhiculent de nombreuses revendications sur le problème des transports, popularisées par le slogan « L'état ne nous transporte pas, il nous roule ». Cette crise débouche sur la mise en place de politiques publiques visant à améliorer le fonctionnement des réseaux, comme le versement transport en 1971 (Lefèvre et Offner, 1990). La période de dix ans encadrée par ces deux dates (1958-1968) est très précisément celle de la constitution d'une ingénierie du trafic à la française qui importe un ensemble de dispositifs statistiques et de méthodes de calcul et de prédiction au traffic engineering américain (Commenges, 2013 ; Dupuy, 1975).

Cette discipline naissante aurait pu s'affranchir du chemin tracé par les études antérieures et par le dispositif de recueil des navettes, mais elle contribue au contraire à accentuer la direction prise dans les études des déplacements en se focalisant sur les actifs occupés. Ce constat peut sembler paradoxal : le corpus méthodologique américain étant importé, il ne s'inscrit a priori pas dans la dépendance au chemin. En fait, ce corpus ne s'intègre pas tel quel dans le paysage technique français, il se greffe sur l'ensemble de connaissances acquises grâce aux navettes, et plus généralement sur les dispositifs mis en place par I'INSEE. Les archives et les entretiens tendent à conforter cette idée. Les documents produits au début des années 1960 montrent une collaboration étroite entre l'ingénierie du trafic (SERC, IAURP) et I'INSEE (Goldberg, 1964 ; SERC, 1966), ce qui est confirmé par les entretiens avec Serge Goldberg, Pierre Merlin et Michel Barbier qui mentionnent la présence permanente d'un technicien détaché de l'INSEE à I'IAURP.

Cette collaboration mène à un renforcement du dispositif de recueil des navettes au recensement de 1962, par l'intermédiaire d'un questionnaire complémentaire sur les transports. Ce questionnaire matérialise la greffe de l'ingénierie du trafic sur les dispositifs mis en place par l'INSEE ${ }^{3}$. II s'agit d'un feuillet de quatre pages adressé à

$3 \quad$ Notre corpus d'archives et d'entretiens ne permet pas de retracer très précisément la genèse de ce questionnaire. Pour Pierre Merlin il a été conçu durant l'année 1961 dans cadre du groupe de travail présidé par Jean Lapébie et dont Serge Goldberg était le rapporteur. Serge Goldberg ne le confirme pas explicitement mais il indique qu'à cette 
tous les actifs occupés du ménage qui renseigne sur les véhicules à disposition, le lieu de travail précis (rue et numéro de rue), les différents moyens de transport utilisés habituellement pour réaliser le déplacement domicile-travail (possibilité de renseigner jusqu'à quatre moyens de transport constituant un déplacement multimodal), les heures de début et de fin de travail, la durée du trajet et le fait de rentrer déjeuner à domicile (Merlin, 1967 : 114). Ce questionnaire complémentaire est à plusieurs titres exceptionnel, il reflète la montée en importance du « problème des transports », donc des navettes, dans le débat public : « quatre pages sur les migrations alternantes ! C'est une grande chance, surtout quand on sait à quel point I'INSEE est, à juste titre, avare du nombre de questions » (Entretien avec P. Merlin). Cette extension du recensement illustre bien la dépendance au chemin : le dispositif statistique originel s'enrichit du point de vue des variables produites mais continue de se limiter à la même fraction de la population, alors qu'il aurait été imaginable d'étendre le dispositif à d'autres catégories de population en se limitant à une information réduite. Ce n'est qu'en 1999 que le recensement élargit la population d'intérêt avec l'introduction d'une question sur la localisation de l'établissement d'enseignement. Les migrations alternantes des scolaires sont pourtant loin d'être résiduelles : en 1999 elles concernent 5,6 millions d'individus, soit près de $10 \%$ de la population métropolitaine ${ }^{4}$.

Les dispositifs d'enquête spécialisés importés des États-Unis par les ingénieurs des Ponts et Chaussées s'inscrivent eux-aussi dans cette dépendance au chemin. La première enquête à domicile sur les déplacements quotidiens est réalisée à Rennes en 1960, en suivant le modèle des enquêtes américaines. La première enquête de ce type réalisée en région parisienne, dite « enquête pilote », date de 1965 (SRERP, 1968). De son côté, le CNAT commandite plusieurs enquêtes sur les déplacements en région parisienne (Bertrand et Hallaire, 1962). Il s'agit de dispositifs ad hoc, qui bénéficient d'une marge de liberté dans la définition de leurs champs sans bouleverser le système de collecte des navettes. Cependant, ils continuent de se focaliser sur les actifs occupés, de deux façons différentes. Soit ils s'intéressent uniquement à cette catégorie de population (cas de l'enquête du CNAT). Soit ils établissent une partition du questionnaire entre un tronc commun, destiné à l'ensemble de la population, et un « questionnaire complémentaire », qui est parfois plus long que le tronc commun et qui ne s'adresse qu'aux actifs occupés (cas de l'enquête pilote de 1965).

Certains facteurs spécifiques à la planification des transports viennent renforcer le monopole des navettes et marquer plus encore la dépendance au chemin. Les méthodes importées des États-Unis visent avant tout à calibrer des infrastructures de transport. Le calibrage se fait sur des critères de dimensionnement: les déplacements domicile-travail sont les déplacements « dimensionnants », l'heure de pointe est I'heure «dimensionnante ». Pierre Merlin résume très clairement cette

époque seul Joseph Elkouby (décédé au moment de mener les entretiens) et lui-même étaient à même de proposer un tel questionnaire.

$4 \quad$ Le calcul est réalisé sur le fichier des mobilités pour étude du recensement de 1999 (France métropolitaine, arrondissements Paris-Lyon-Marseille désagrégés). Source : INSEE (producteur), CMH (diffuseur), Recensement de la population 1999, tableaux "Mobilités". 
approche : les migrations alternantes présentent un intérêt indéniable parce qu'elles constituent l'essentiel des déplacements effectués à l'heure de pointe et que c'est le nombre de ceux-ci qui entraîne la congestion du réseau de circulation parisien et qui conditionne l'ampleur des aménagements nécessaires (Merlin, 1967 : 113). Les précisions apportées par Merlin à ce propos sont éclairantes : il insiste sur le fait qu'il n'entend pas négliger les déplacements autres, que ces déplacements « constituent, globalement, la majeure partie de la demande de transports urbains » (p. 263), mais que cette demande n'intervient que marginalement sur la capacité à donner aux réseaux. Les déplacements autres constituent donc une majorité silencieuse qui doit laisser place aux navettes pour des impératifs de planification des transports.

La socio-économie des transports s'inscrit dans un cadre plus ancien qui se focalise sur la population active occupée. Celle-ci fait l'objet, dès la fin du XIXe siècle, d'un travail de définition qui tend à la caractériser finement en rejetant le reste de la population dans des catégories résiduelles. Le terme de « population active occupée » apparaît en effet avec le recensement de 1896, en négatif de la catégorie de chômeur et de la catégorie d'inactif (Fouquet, 2004). C'est l'origine de la partition fondamentale de la population en deux catégories : actifs et inactifs. La première fait l'objet d'un effort constant de caractérisation statistique et comptable (Vanoli, 2002), l'autre est considérée comme « oisive » (SGF, 1899). La socioéconomie des transports renforce ce trait : formés dans les universités américaines à la recherche opérationnelle, les ingénieurs du Corps et Ponts et Chaussées vont se charger d'optimiser la ville conçue comme système productif. Dans ce cadre, toute l'attention est portée sur la population active. L'analyse marxiste, si elle est réductrice, n'en est pas moins éclairante : la planification des transports peut être considérée comme l'optimisation d'un système de mobilisation de la main d'œuvre (Bonnet 1978).

\section{L'urbain saisi par les navettes}

A la fin des années 1950, le discours sur le déséquilibre occupe le devant de la scène et ses conséquences néfastes sont mises en avant. En ce qui concerne les liens entre communes, c'est la saturation des réseaux de transport, en ce qui concerne les espaces, c'est l'inégale répartition des ressources fiscales. Le discours sur le déséquilibre est ainsi à l'origine des premières tentatives de zonage fonctionnel en France ${ }^{5}$ : « pour rétablir l'équilibre, au moins approximativement, le ministère de l'Intérieur a pris un décret du 28 mars 1957 ; l'article premier de ce décret pose le principe de la constitution d'“agglomérations": "Pour l'application du présent décret, les communes dont une partie importante de la population active travaille en dehors du territoire communal et qui se trouvent privées de ressources

$5 \quad$ L'apparition des zonages fonctionnels s'inscrit dans la lignée, bien plus ancienne, des tentatives de définition de la ville. Plusieurs travaux ont recensé ces tentatives, dont les plus anciennes datent du XIXe siècle, et montrent l'utilisation d'une variété de critères définitionnels: seuils de population, critères morphologiques, courbes isochrones, etc. (Cattan et al., 1994 ; Le Gléau et al., 1996 ; Bretagnolle, 2009). 
normales, seront incluses dans des agglomérations comprenant la ou les communes attractives" ». Le zonage est d'abord réalisé en région parisienne : « le ministère de l'Intérieur a demandé à I'INSEE de lui délimiter en premier lieu l'“agglomération parisienne" où se trouvent les cas les plus alarmants de communes dortoirs » (Brichler, 1958 : 102-103). La méthode utilisée s'appuie sur plusieurs variables de population et d'emploi ainsi que sur les navettes. II n'est pas encore question de mesurer des liens intermunicipaux, mais seulement des soldes : le pourcentage de pertes journalières de population active occupée (Brichler et Bastié, 1960 ; Bertrand et Hallaire, 1962).

Au début des années 1970, I'INSEE se lance dans la construction d'une méthode de zonage plus élaborée, fondée non plus sur des soldes mais sur des liens intermunicipaux. II s'agit de créer des bassins d'emploi pour établir des mesures cohérentes des taux d'emploi et de chômage malgré le déséquilibre des lieux de résidence et d'emploi. Christophe Terrier (1980) se lance dans la réalisation d'une Méthode Informatique de Recherche et d'Analyse des Bassins par l'Étude des Liaisons Logement-Emploi (MIRABELLE). Cette nouvelle étape illustre bien le rôle assigné à la mobilité quotidienne, et plus particulièrement aux navettes pour appréhender la structuration de l'espace. Quand le dispositif statistique cherche à produire des variables de stock correspondant à un découpage d'unités spatiales, la mobilité entre unités spatiales pose problème. Quelle que soit la variable d'intérêt, il y a toujours une question en suspens : où doit être compté un individu présent, à un titre quelconque, dans plusieurs unités spatiales ? Cette question ramène encore une fois la mobilité à un rôle subalterne, elle n'est pas un objet d'étude en soi, mais le moyen de constituer une mesure plus cohérente d'autre chose. Les remarques de Christophe Terrier sur son travail de zonage au début des années 1970 sont éclairantes : " l'idée était en fait de nier la mobilité, c'est-à-dire de l'enfermer dans un espace où on ait à la fois les deux bouts de la chaîne, le domicile et le travail. Ça a toujours été ça la démarche bassin d'emploi, parce qu'on ne peut pas analyser un marché de l'emploi où on a le domicile dans un territoire et le travail dans un autre territoire. Le taux de chômage est toujours rapporté au lieu de domicile et le taux d'activité au lieu d'emploi. Donc on il fallait unir les deux, pour que l'essentiel des mouvements s'inscrivent à l'intérieur du même territoire » (Entretien avec Ch. Terrier). Plus loin dans l'entretien, il insiste sur l'objectif de la constitution de bassins d'emploi : « on m'avait demandé de faire une méthode de détermination des bassins d'emploi, c'est-à-dire d'enfermer les déplacements domicile-travail ».

Au début des années 1990, le zonage en ZPIU est devenu inopérant (Le Jeannic, 1996), et I'INSEE engage un processus de révision qui aboutit en 1996 à un nouveau zonage: le Zonage en Aires Urbaines (ZAU). Les archives conservées par Mohamed Hilal contiennent un ensemble de notes autour de cette révision provenant de la plupart des directions régionales de I'INSEE. L'analyse de ce corpus laisse apparaître des discussions nourries qui portent sur l'objet et/ou l'objectif du nouveau zonage, la méthode à utiliser et les données sur lesquelles s'appuyer.

Les discussions sur l'objet et sur l'objectif du nouveau zonage méritent toute notre attention car elles laissent apparaître un vaste éventail de possibilités et d'incertitudes. « Nous sommes de plus en plus sollicités pour définir des territoires pertinents au regard de certaines problématiques : le maintien et le développement 
des services à la population (bassin de vie) ; le devenir des espaces "ruraux" confrontés à la déprise agricole (espace "délaissé"); la relation entre les villes et leur environnement périurbain (bassin d'habitat, transports et infrastructures); les bassins d'emploi constitués autour des principaux pôles d'une zone d'emploí ${ }^{\prime}$. Pour la plupart des experts des directions régionales de l'INSEE, les zones à créer seront des bassins, mais rien n'est fixé concernant le contenu conceptuel de ces bassins : bassins de vie, d'emplois, d'habitat, etc. "S'agit-il de mettre au point un instrument tourné principalement vers la description de l'espace territorial, des unités administratives et lesquelles, de la population ou de l'activité ? [...] quel est

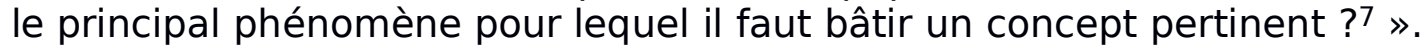

Les experts de I'INSEE insistent à plusieurs reprises sur les multiples objets possibles et les confusions sur le contenu des zonages, confusions existantes ou à éviter. La première est la confusion entre zone urbaine et bassin d'emploi : « on pourra ainsi [grâce au nouveau zonage] lever l'ambiguïté qui découle en fait d'une confusion souvent implicite, chez certains utilisateurs, entre le concept de ZPIU et celui de zone d'emploi8 ${ }^{»}$. Peut-on par un même procédé à la fois délimiter des bassins d'emploi et distinguer l'urbain du rural : « on pourrait profiter de l'occasion pour redéfinir la distinction entre communes urbaines et rurales ${ }^{9} »$. La question posée, celle de la polysémie du zonage, est bien résumée par Philippe Julien : « il transparaît que l'on s'est, jusqu'à présent, plus interrogé sur le QUOI (remplacer les ZPIU), voire sur le COMMENT que sur le POURQUOI (si ce n'est en disant que les ZPIU ne convenaient plus). Or nous savons tous que [...] à chaque problématique spatiale devrait correspondre un zonage particulier ${ }^{10} »$. Le deuxième élément de polysémie concerne la fusion des notions de bassin d'emploi et de bassin de vie. Celle-ci est en germe quinze ans plus tôt dans le texte de Christophe Terrier (1980) qui présente la méthode MIRABELLE. Le nom de la méthode, « méthode de recherche des bassins », ne précise pas de quels bassins il s'agit. Dans le texte il est presque toujours question de bassins d'emploi, mais cette notion est parfois étendue à la notion de «bassins socio-culturels » qui préfigure celle de bassin de vie.

Concernant la méthode, l'approche fonctionnelle ou relationnelle, fondée sur des liens intermunicipaux, semble emporter l'accord de toutes les personnes impliquées. Deux questions font débat : I'algorithme d'agrégation à utiliser ${ }^{11}$ et la question des seuils. Doit-on fixer des pôles a priori à partir d'un seuil, et doit-il s'agir d'un seuil de

$6 \quad$ M. Trégouet, INSEE-DR Aquitaine, Note sur la révision des ZPIU, 8 novembre 1993.

7 J.-L. Heller, INSEE-DR Languedoc-Roussillon, Note sur la révision des ZPIU, 17 novembre 1993.

8 J.-J. Ronsac, INSEE-DR Île-de-France, Note sur la révision des ZPIU, 2 novembre 1993.

9 J.-J. Ronsac, INSEE-DR Île-de-France, Note sur la révision des ZPIU, 2 novembre 1993.

10 Ph. Julien, INSEE-DR Midi-Pyrénées, Note sur la révision des ZPIU, 4 novembre 1993.

11 Cette question fait débat essentiellement parce que deux des personnes du groupe de travail, Christophe Terrier et Loeiz Laurent, ont chacun développé un algorithme pour cela, respectivement «MIRABELLE » et « Zonages ». 
population ou d'un seuil d'emplois ? Ces débats, très intéressants, sont extérieurs à notre propos.

Concernant les données à utiliser, le consensus est frappant, il apparaît aussi bien dans les notes et comptes-rendus de réunion que dans les entretiens : Pierre Audibert affirme que « la délimitation de la zone périurbaine doit s'appuyer uniquement sur l'analyse des flux domicile-travail. Les migrations alternantes constituent le seul indicateur d'interdépendance entre l'urbain et le périurbain qui soit connu de façon simple, objective et exhaustive ${ }^{12} »$. Thomas Le Jeannic explique : « la mesure la plus évidente c'était les navettes domicile-travail données par le recensement. II y a eu une première discussion sur "quelles sont les sources qui nous permettent d'élaborer cette influence des villes ?" et au bout de quelques réunions on a compris que la base ce serait les migrations alternantes du recensement de la population. Point barre. Donc source unique ${ }^{13} \gg$. La possibilité d'utiliser un dispositif ad hoc de mesure de la mobilité est rapidement écartée (cf. Section 2, citation de M. Hilal), la possibilité d'étendre le dispositif de recueil des navettes à une partie plus vaste de la population n'est pas envisagée (du moins dans les documents à notre disposition), cependant il est proposé d'ajouter une question complémentaire pour renforcer encore le dispositif de mesure des navettes : « il est évident que la migration alternante est une variable plus que pertinente. Il faudrait arriver à la débarrasser des scories liées aux fausses migrations quotidiennes. Question au RP ? $^{14}$ ».

Finalement, le zonage en aires urbaines élaboré durant les années 1990 est une illustration frappante de la dépendance au chemin. Plusieurs méthodes ont été testées, des débats ont eu lieu au sein du groupe pour l'utilisation de programmes de zonage préexistants (MIRABELLE, Zonages), la question de la lisibilité du processus et du résultat du zonage a été posée à maintes reprises. La question de l'objet du zonage montre un vaste éventail de possibilités : doit-on faire un seul zonage ou bien plusieurs zonages répondant à différentes problématiques ? Que doit montrer le zonage: des zones urbaines, des bassins d'emploi, des bassins de vie ? Or, tout au long de ce processus à méthode et objet variables, le fait d'utiliser les navettes comme source unique n'a jamais été remis en cause. Ce monopole est d'autant plus remarquable qu'il y avait dans d'autres pays plusieurs exemples de zonage multicritères, et que les données du recensement français de 1968 ont elles-mêmes été utilisées par des chercheurs anglo-saxons dans une approche multicritère (Hall et Hay, 1980). Ceci étant dit, les approches alternatives n'apparaissent que dans des travaux de recherche. Les instituts de statistique officiels, aux Etats-Unis et au Royaume-Uni par exemple, ne se distinguent par de l'approche française. Comme en France, les zonages fonctionnels définissent à la fois de l'urbain et du bassin d'emploi (standard metropolitan areas aux Etats-Unis,

12 P. Audibert, INSEE-DR Rhônes-Alpes, Note sur la révision des ZPIU, 15 novembre 1993.

13 Entretien avec Thomas Le Jeannic.

14 M. Roussel, INSEE-DR Poitou-Charentes, Note sur la révision des ZPIU, 4 novembre 1993. 
travel-to-work areas au Royaume-Uni). Comme en France, la méthode repose sur les navettes domicile-travail (Coombes et al., 1986 ; OMB, 1998 ; 2000).

Après soixante ans de montée en importance dans le paysage technique et scientifique, l'objet navette est devenu incontournable. Il est porteur d'une longue tradition d'études qui ont contribué à façonner les notions clefs de l'analyse des territoires (zone urbaine - bassin d'emploi - bassin de vie). En 2010, I'INSEE réalise un nouveau zonage en aires urbaines (Brutel, 2011) qui s'inscrit pleinement dans la continuité de celui des années 1990, confortant ainsi le rôle structurant donné aux navettes.

\section{Conclusion}

La construction et la consolidation de la société salariale s'appuie sur une certaine définition du travail (exercice d'une activité rémunérée), de la population active occupée (celle qui exerce un travail rémunéré) et par une distinction nette entre le monde du travail et le monde domestique (distinction entre les lieux et les liens appartenant à ces deux mondes). Quand Antoine Haumont (2000) parlait de « la mobilité quotidienne dans une société salariale », il se référait aux caractéristiques particulières de la mobilité dans une société de ce type. Nous avons ici mis en avant la façon dont une telle société salariale construit une connaissance sur elle-même, en particulier grâce à des dispositifs statistiques. D'une part, ces dispositifs ne sont pas de simples instruments de mesure extérieurs à la conceptualisation des phénomènes. D'autre part, ils ne fonctionnent pas de façon libre et linéaire, avec un reset à chaque nouvelle étape. Ils sont au contraire dépendants au chemin, et suivent un processus auto-entretenu de production et d'utilisation. L'expression d'Antoine Haumont revêt dès lors un double sens : elle désigne les caractéristiques particulières de la mobilité quotidienne dans le cadre d'une société salariale ; elle désigne également une certaine vision de la mobilité quotidienne façonnée par les dispositifs statistiques de cette société salariale. En d'autres termes, c'est l'avènement d'une société salariale qui produit les navettes : à la fois le mot (I'objet statistique) et la chose (les flux de déplacements domicile-travail).

Les travaux visant à délimiter et qualifier des espaces se caractérisent, dans le contexte technique et scientifique français, par un rapport différencié à la théorie et à l'empirie. Cependant, l'ensemble des études et des recherches s'appuyant sur les navettes véhiculent, de façon implicite ou bien de façon explicite et théorisée, une vision économiste des rapports sociétés-espaces. Nous ne cherchons pas ici à porter un jugement sur l'idée, formulée comme une hypothèse, comme un postulat ou comme un résultat, que l'organisation spatiale du travail est le vecteur primordial de structuration de l'espace. Nous voulons simplement montrer que cette idée est le résultat d'un processus auto-entretenu, vieux de plus d'un demisiècle, de co-construction de dispositifs de production de données, de pratiques institutionnelles et de discours politique, technique et scientifique. 


\section{Bibliographie}

Affichard, J. (dir.) (1977a), Pour une histoire de la statistique, vol.1, Paris, INSEE, 593 p.

Affichard, J. (dir.) (1977b), Pour une histoire de la statistique, vol.2, Paris, INSEE, $969 \mathrm{p}$.

Allain, J.-M. (2007), « La mixité sociale à l'épreuve des nouvelles temporalités », Espace Populations Sociétés, 2-3, p. 299-304.

Arrow, K. (2000), « Increasing returns: historiographic issues and path dependence », The European Journal of the History of Economic Thought, 7, p. 171180.

Berger, M. (2004), Les périurbains de Paris. De la ville dense à la métropole éclatée? Paris, CNRS Éditions, $317 \mathrm{p}$.

Berroir, S. (1998), Concentration et polarisation: vers une nouvelle organisation des espaces urbanisés, Thèse de doctorat, Université de Paris I, 399 p.

Berroir, S., Cattan, N., Giraud, Th., Saint-Julien, Th. (2007), Fonctionnement métropolitain du Bassin parisien. Une analyse de la mobilité des populations par les $D A D S$, Étude réalisée pour la DIACT, 108 p.

Berroir, S., Mathian, H., Saint-Julien, Th., Sanders, L. (2004), Mobilités et polarisations : vers des métropoles polycentriques. Le cas des métropoles francilienne et méditerranéenne, Étude réalisée pour le PUCA, 145 p.

Bertrand, P., Hallaire, J. (1962), « Une enquête sur les déplacements journaliers de la population active de la Région Parisienne », Journal de la Société de Statistique de Paris, 7-8-9, p. 186-217.

Bretagnolle, A. (2009), Villes et réseaux de transport. Des interactions dans la longue durée, Habilitation à diriger des recherches, vol.3, Université Paris I Panthéon Sorbonne, 204 p.

Brichler, M. (1958), « Méthode de recherche utilisée pour la détermination des communes dortoirs de la région parisienne », Journal de la Société de Statistique de Paris, 4-5-6, p. 96-118.

Brichler, M., Bastié, J. (1960), « Délimitation de l'agglomération parisienne », Population, 15, p. 433-456.

Brutel, Ch. (2011), « Le nouveau zonage en aires urbaines de 2010 », INSEE Première, 1374, $4 \mathrm{p}$.

Bunle, H. (1932), «Migrations alternantes dans la région parisienne », Bulletin de la Statistique Générale de la France, 21, p. 585-612.

Coombes, M.G., Green, A.E, Openshaw, S. (1986) « An efficient algorithm to generate official statistical reporting areas: the case of the 1984 travel-to-work areas in Britain », Journal of the Operational Research Society, vol.37, n-10, pp. 943-953. 
Comité National pour l'Aménagement des Temps de Travail (1964), Le temps de mieux vivre, Paris, CNAT, 9 p.

Cattan, N., Pumain, D., Rozenblat, C., Saint-Julien, Th. (1994), Le système des villes européennes, Paris, Anthropos, 201 p.

Chevalier, L. (1956), «La statistique et la description sociale de Paris », Population, 4, p. 621-652.

Claval, P. (1980), Les mythes fondateurs des sciences sociales, Paris, Presses universitaires de France, $261 \mathrm{p}$.

Cohen, E. (2004), « Expliquer Paris à la télévision : Pierre Sudreau et les problèmes de la construction (1958) », Sociétés et Représentations, 17, p. 117-127.

Commenges, H. (2013), L'invention de la mobilité quotidienne. Aspects performatifs des instruments de la socio-économie des transports, Thèse de doctorat, Université Paris Diderot, $391 \mathrm{p}$.

Courgeau, D. (1988), Méthodes de mesure de la mobilité spatiale : migrations internes, mobilité temporaire, navettes, Paris, INED, $301 \mathrm{p}$.

Desrosières, A. (2003), « Historiciser l'action publique : l'État, le marché et les statistiques », in Laborier, P., Trom, D. (dir.), Historicités de l'action publique, Paris, Presses universitaires de France, p. 207-221.

Desrosières, A., Thévenot, L. (1979), «Les mots et les chiffres : les nomenclatures socio-professionnelles », Economie et statistique, 110, p. 49-65.

Desrosières, A., Thévenot, L. (1988), Les catégories socioprofessionnelles, Paris, La Découverte, $125 \mathrm{p}$.

Didier, E. (2009), En quoi consiste l'Amérique ? Les statistiques, le New deal et la démocratie, Paris, La Découverte, 317 p.

Dupuy, G. (1975), Une technique de planification au service de l'automobile. Les modèles de trafic urbain, Paris, Ministère de l'équipement, 201 p.

Flaus, L. (1953), « Lieux de travail et zones d'habitation dans le département de la Seine », Journal de la Société de Statistique de Paris, 4-5-6, p. 98-124.

Fouquet, A. (2004), « L'invention de l'inactivité », Travail, genre et sociétés, 11, p. 47-62.

Gardon, S. (2009), Gouverner la circulation urbaine : des villes françaises face à I'automobile, Thèse de doctorat, Institut d'Études Politiques de Lyon, 580 p.

Gardon, S., Passalacqua A., Schipper F. (2009), « Pour une histoire des circulations sur la circulation », Métropoles, 6, p. 1-12.

Gilli, F. (2002), « Déplacements domicile-travail et organisation du Bassin parisien », L'Espace géographique, 31, p. 289-305.

Godinot, A. (2005), «Pour comprendre le recensement de la population », INSEE Méthodes, Hors série, $\mathrm{np}$. 
Goldberg, S. (1964), Les études globales de transport. Cours de recyclage consacré à l'étude de la circulation urbaine, Cours de recyclage, Paris, Ecole Nationale des Ponts et Chaussées, $17 \mathrm{p}$.

Gravier, J.-F. (1947), Paris et le désert français : décentralisation, équipement, population, Paris, Le Portulan, $412 \mathrm{p}$.

Haumont, A. (2000), « Mobilité quotidienne dans la société salariale », in Bonnet M., Desjeux D. (dir.), Les territoires de la mobilité, Paris, Presses Universitaires de France, $225 \mathrm{p}$.

Hilal, M., Piguet, V., Schmitt, B. (1995), « Communes rurales et petites villes dans la hiérarchie urbaine », Économie et Statistique, 282, p. 21-36.

Hilal, M., Sencebe, Y. (2003), Analyse des processus d'ancrage et des formes de territorialité des populations marquées par l'éclatement de leurs lieux de travail et de domicile à la périphérie des pôles urbains, Programme « Mobilité et territoires urbains », Centre d'économie et de sociologie appliquées à l'agriculture et aux espaces ruraux, $204 \mathrm{p}$.

Institut d'Aménagement et d'Urbanisme de la Région Parisienne (1966), Cahiers de I'IAURP, vol.4-5, Paris, IAURP.

Institut National de la Statistique et des Études Économiques (1998), «Les zonages : enjeux et méthodes », INSEE Méthodes, 83, $132 \mathrm{p}$.

Institut National de la Statistique et des Études Économiques, Institut National de la Recherche Agronomique, Délégation à l'Aménagement du Territoire et à I'Action Régionale (2002), Carte des territoires vécus, INSEE, IGN, Cartographie et Décision.

Julien, Ph. (2000), « Mesurer un univers urbain en expansion », Économie et statistique, 336, p. 3-33.

Kain, J.F. (1968), « Housing segregation, negro employment, and metropolitan decentralization », The Quarterly Journal of Economics, 82, p. 175-197.

Kain, J.F. (2004), «A pioneer's perspective on the spatial mismatch literature », Urban Studies, 41, p. 7-32.

Le Corbusier (1971), La Charte d'Athènes, Paris, Editions du Seuil, 189 p.

Le Gléau, J.-P., Pumain, D., Saint-Julien, Th. (1996), « Villes d'Europe : à chaque pays sa définition », Économie et statistique, 294-295, p. 9-23.

Le Jeannic, Th. (1996), «Une nouvelle approche territoriale de la ville », Économie et statistique, 294-295, p. 25-45.

Maquet, H. (1963), L'aménagement des horaires de travail dans le cadre de la journée. Paris, CNAT, $175 \mathrm{p}$.

Massot, M.-H. (1998), «Espace de vie, espaces de travail », in Pumain D., Mattei M.F. (dir.), Données urbaines, vol.2, Paris, Anthropos, p.147-156.

Massé, P. (1965), Le plan ou I'anti-hasard, Paris, Gallimard, 250 p.

Merlin, P. (1967), Les transports parisiens : étude de géographie économique et sociale, Paris, Masson, $495 \mathrm{p}$ 
Moret-Lespinet, I. (2006), «La création du ministère du Travail », Actes du colloque « Les questions sociales au parlement (1789-2006) », Paris, Comité d'histoire parlementaire et politique.

Office of Management and Budget (1998) « Alternative approaches to defining metropolitan and nonmetropolitan areas », Federal Register, vol.63, n 244.

Orfeuil, J.-P. (1996), «La mobilité urbaine, son coût, ses modalités de financement », in Données urbaines, in Pumain, D., Godard, F. (dir.), Données urbaines, vol.1, Paris, Anthropos, p. 187-198.

Pierson, P. (2000), "Increasing returns, path dependence, and the study of politics », American Political Science Review, 94, p. 251-267.

Service du Recensement (1904), Résultats statistiques du recensement général de la population de 1901, vol. 1, Paris, Service du Recensement.

Société d'Économie et de Mathématiques Appliquées (1963), « Etude des déplacements domicile-travail. Le modèle d'équilibre préférentiel », Paris, SEMA, 38 p.

Service d'Études et de Recherches sur la Circulation (1966), Analyse des déplacements domicile-travail. Étude comparative de quelques villes françaises, Paris, SERC, $211 \mathrm{p}$.

Service Régional de l'Équipement de la Région Parisienne (1968), Enquête pilote 1965 sur les déplacements en région parisienne, Maisons-Alfort, SRERP.

Schor, P. (2001), Compter et classer : histoire des catégories de la population dans le recensement américain, 1790-1940, Thèse de doctorat, Ecole des Hautes Etudes en Sciences Sociales, 515 p.

Statistique Générale de la France (1899), Résultats statistiques du dénombrement de 1896, Paris, Statistique Générale de la France.

Talbot, J. (2001), « Les déplacements domicile-travail. De plus en plus d'actifs travaillent loin de chez eux », INSEE Première, 767, p. 1-4.

Terrier, Ch. (1980), « Les structures de l'espace français par les migrations alternantes », Actes du colloque «Migrations internes et externes en Europe Occidentale », Lille, Université des Sciences et Techniques de Lille.

Vallès, V., Hugon, P. (1994), «Une approche des bassins de vie. L'influence des villes et des bourgs-centres », INSEE Première, 325, p.1-4.

Vanoli, A. (2002), Une histoire de la comptabilité nationale, Paris, La Découverte, $655 \mathrm{p}$.

Vatin, F., Bernard, S. (2007), Le salariat : théorie, histoire et formes, Paris, La Dispute, $346 \mathrm{p}$.

Volle, M. (1982), Histoire de la statistique industrielle, Paris, Economica, 302 p.

Wallerstein, I. (2009), Comprendre le monde. Introduction à l'analyse des systèmesmonde, Paris, La Découverte, 173 p. 